\title{
MOBILIDADE DE METAIS PESADOS EM SOLO TRATADO COM RESÍDUO SIDERÚRGICO ÁCIDO(1)
}

\author{
N. M. B. AMARAL SOBRINHO ${ }^{(2,5)}$, A. C.X. VELLOSO ${ }^{(3,5)}$, \\ L. M. $\operatorname{COSTA}^{(4,5)} \&$ C. OLIVEIRA ${ }^{(2)}$
}

\begin{abstract}
RESUMO
Amostras do horizonte Bt de um Podzólico Vermelho-Amarelo (PV), predominante no aterro-piloto de resíduos industriais (ARSI) da Companhia Siderúrgica Nacional (CSN), foram acondicionadas, em 1992, em colunas de vidro com $5,4 \mathrm{~cm}$ de diâmetro e $37 \mathrm{~cm}$ de altura. Sobre essas amostras de solo foram colocadas amostras de um resíduo ácido da CSN na proporção solo-resíduo de 4:1 (conforme a concepção básica do projeto do ARSI). Tal sistema resíduo-solo foi lixiviado com 50 volumes-poro de água deionizada, com pH 4,5, com o objetivo de avaliar a movimentação de $\mathrm{Cr}, \mathrm{Mn}, \mathrm{Ni}, \mathrm{Cu}, \mathrm{Zn}, \mathrm{Cd}$ e $\mathrm{Pb}$ nas colunas. Depois da lixiviação, removeu-se o resíduo do topo das colunas, e seccionaram-se as amostras de solo em 4 partes iguais. Os metais pesados das amostras do resíduo e do solo for am extraídos seqüencialmente. $\mathrm{OPb}, \mathrm{Cu}$ e $\mathrm{Cr}$ mobi lizados do resíduo ficaram retidos nos primeiros $5 \mathrm{~cm}$ da coluna de solo. $0 \mathrm{Zn}$, Mn, Ni e Cd tiveram aumento da concentração em profundidade. Nos efluentes das colunas com o resíduo, foram observadas concentrações de $\mathrm{Mn}$ e $\mathrm{Ni}$ bem superiores aos padrões permitidos pela Fundação Estadual de Engenharia do Meio Ambiente (FE EMA, 1985).
\end{abstract}

Termos de indexação: metal pesado, resíduo siderúrgico, mobilidade iônica.

\footnotetext{
(1) Parte da Tese de Doutorado do primeiro autor, apresentada ao Departamento de Solos da Universidade Federal de Viçosa-UFV, Viçosa (MG). Pesquisa realizada com recursos da Companhia Siderúrgica Nacional (CSN). Recebido para publicação em setembro de 1996 e aprovado em março de 1998.

(2) Professor do Departamento de Solos, Universidade Federal Rural do Rio de J aneiro-UFRRJ. CEP 23851-970 Seropédica (RJ ).

(3) Professor do Laboratório de Solos do Centro de Ciências e Tecnologia Agropecuárias, CCTA, Universidade Estadual do Norte Fluminense-UENF. Av. Alberto Lamego nํ2 2000, CEP 28015-620 Campos dos Goytacazes (RJ ).

(4) Professor do Departamento de Solos, Universidade Federal de Viçosa-UFV. CEP 36570-000 Viçosa (MG).

${ }^{(5)}$ Bolsista do CNPq.
} 


\title{
SUMMARY: HEAVY METAL MOBILITY IN SOIL COLUMNS TREATED WITH AN ACID IRON SMELTER RESIDUE
}

\begin{abstract}
Air dried subsurfacesamples of a heavy textured Red-Yel low Podzolic soil, representative of theindustrial solid residuepilot landfill (ISRL) of the National Sted Works (Companhia Siderúrgica Nacional-CSN), were packed in $5.4 \mathrm{~cm}$ width and $37 \mathrm{~cm}$ high gl ass col umns. An acid iron smelter residue was placed on the top of the soil columns to make a 4:1 soilresidue ratio, in consonance with the basic CSN project. To eval uate $\mathrm{Cr}, \mathrm{Mn}, \mathrm{Ni}, \mathrm{Cu}, \mathrm{Zn}, \mathrm{Cd}$ and $\mathrm{Pb}$ mobilities, the system soil-residue was leached with 50 pore vol umes of dei onized water at $\mathrm{pH}$ 4.5. After leaching, theresiduewas removed from thetops and thesoil columns were sectioned into 4 equal parts. The heavy metals in the residue and in the soil samples were sequentially extracted. $\mathrm{Pb}, \mathrm{Cr}$ and $\mathrm{Cu}$ dissolved from theresi dues were retained in the upper 0-5 cm soil columns. $\mathrm{Zn}, \mathrm{Mn}, \mathrm{Ni}$ and $\mathrm{Cd}$ showed the same distribution pattern, i.e, increased concentration with depth. In the effluents leaching out the columns (with the residue on the top) theleve $\mathrm{s}$ of $\mathrm{Mn}$ and $\mathrm{Ni}$ werehigher than thestandard limits all owed by FEEMA (1985).
\end{abstract}

Index terms: heavy metal, steel works residue, landfill, leaching, metal mobility.

\section{INTRODUÇÃO}

De modo geral, os resíduos resultam de atividades industriais ou desistemas de tratamento de diversas origens, podendo apresentar-se nas formas sólida, semi-sólida, líquida ou gasosa. Em função de suas características específicas, podem apresentar risco efetivo ou potencial à saúde humana, ou gerar impactos aos meios físico, biótico e sócio-econômico, exigindo cuidados especiais quanto ao manuseio, acondicionamento, coleta, transporte e disposição final. Tal disposição depende do grau de periculosidade do resíduo, podendo acontecer em aterros convencionais, para os resíduos inertes, eem aterros especiais, para os não-inertes e/ou perigosos.

A CSN vem desenvolvendo projetos para a disposição adequada dos seus resíduos no ARSI. Nesses estudos, têm si do obtidas informações básicas sobre as características físicas e químicas dos resíduos (COBRAPI, 1986) e os prováveis riscos de contaminação desol os eáguas subterrâneas (Amaral Sobrinho, 1993). A geração específica dos resíduos sólidos da CSN é de $634 \mathrm{~kg} \mathrm{t}^{-1}$ de aço líquido produzido (Cantini \& Souza, 1989). Dos resíduos gerados de $2.700 .000 \mathrm{t}$ ano-1, 4,9\% deverão ser dispostos no ARSI. A possível contaminação por metais pesados dos aqüíferos próximos ao ARSI dependerá, dentre numerosos fatores, da concentração e solubilidade dos metais pesados presentes nos resíduos, da quantidade de resíduo colocada no aterro, da precipitação pluviométrica local e da capacidade de interação desses resíduos com a fase sól ida do solo (inorgânica e/ou orgânica). Essa interação é bastante complexa, envolvendo reações de adsor ção/dessor ção, precipitação/ dissolução, complexação e oxirredução.
O movimento dos metais pesados no solo tem sido objeto de numerosas pesquisas (Biddappa et al., 1982; K han \& Frankland, 1983; Williams et al., 1985; Welch \& Lund, 1989; Alloway, 1990).Tais estudos têm demonstrado que os metais $\mathrm{Pb}, \mathrm{Cr}$ e Cu apresentam baixa mobilidade, acumulando-se na superfície dos solos contaminados, enquanto $\mathrm{Zn}, \mathrm{Mn}, \mathrm{Ni}$ e, principalmente, $C d$ são relativamente mais móveis, apresentando mai or risco de contaminação da água subterrânea (Sheppard \& Thibault, 1992). No Brasil, os trabal hos dessa natureza são poucos.

Estetrabalho teve por objetivo aval iar, em colunas de amostras de terra, a mobilidade de al guns metais pesados provenientes do resíduo siderúrgico ácido da CSN.

\section{MATERIAL E MÉTODOS}

Três resíduos ácidos, oriundos detratamento com $\mathrm{H}_{2} \mathrm{SO}_{4}$, todos sól idos, produzidos na CSN, localizada no município de Volta Redonda (RJ ), foram col etados nos locais de geração, retirando-se várias amostras simples do topo, meio e da base das pil has de rejeitos, para formar a amostra composta (ABNT,1985). Em seguida, as três amostras compostas foram secas ao ar, destorroadas, quando necessário, com rolo de madeira, e passadas em peneira de plástico de 0,2 mm deabertura aproximadamente. Misturaramse e homogeneizaram-se massas iguais dos três resíduos ácidos. Neste trabalho, tal mistura de resíduos será chamada de resíduo ácido. A mistura apresentou $950 \mathrm{mg} \mathrm{kg}^{-1}$ de sulfato solúvel, $\mathrm{pH} \mathrm{3,8} \mathrm{e}$ os seguintes teores de metais pesados em $\mathrm{mg} \mathrm{kg}^{-1}$ : Mn 13766,00; Ni 1575,00; Pb 3600,00; Cr 873,33; Zn 840,00; Cu 46,08 eCd 5,50. 
Procurou-se simular o aterro-piloto de resíduos industriais (ARSI) da Companhia Siderúrgica Nacional (CSN), utilizando amostras de solo coletadas do horizonte Bt do PV típico do ARSI, acondicionadas em tubos de vidro com $5,4 \mathrm{~cm}$ de diâmetro e $37 \mathrm{~cm}$ de altura, sobre as quais foram col ocadas amostras de resíduo siderúrgico ácido da CSN, de maneira que a proporção altura da coluna de terra para a altura da camada de resíduo fosse de 4:1, aproximadamente, conforme a concepção básica do referido projeto (COBRAPI, 1986). As amostras do Bt do PV típico do ARSI apresentaram $650 \mathrm{~g} \mathrm{~kg}^{-1}$ de argila, $160 \mathrm{~g} \mathrm{~kg}^{-1}$ de silte, $190 \mathrm{~g} \mathrm{~kg}^{-1} \mathrm{de}$ areia, $6,6 \mathrm{~g} \mathrm{~kg}^{-1}$ de carbono orgânico, $130 \mathrm{mmol}_{\mathrm{C}} \mathrm{dm}^{-3}$ de CTC efetiva, pH 4,6 e os seguintes teores de metais pesados em $\mathrm{mg} \mathrm{kg}^{-1}$ : Zn 23; Cu 4,4; Ni 15,0; $\mathrm{Cd}$ I,0; Pb 15,0 e Mn 67,5. 0 ensaio com 5 repetições consistiu em dois tratamentos (testemunha eresíduo ácido) e num total de 10 unidades experimentais (colunas).

\section{Acondicionamento das colunas}

Sobre um disco de vidro perfurado, Iocal izado na parte inferior das colunas, colocou-se uma camada de lã de vidro. A seguir, por meio de um funil de cano longo, que era mantido em contínua movimentação, adicionaram-se, a cada coluna, $600 \mathrm{~g}$ das amostras de solo, quantidade calculada para produzir uma coluna de $20 \mathrm{~cm}$ de altura, aproximadamente. Para obter um procedimento que pudesse ser repeti do uni formemente, além detornar a densi dade da amostra mais regular, as col unas com a quantidade da amostra eram lançadas, através de um suporte, dez vezes (número previamente ajustado) de uma altura de $15 \mathrm{~cm}$ e amortecidas em borracha maciça de $3 \mathrm{~cm}$ de altura.

\section{Determi nação do volume-poro}

Depois do acondicionamento, colocaram-se as colunas em caixas de isopor com água deionizada em quantidade necessária para o aparecimento de uma película deágua no topo da coluna. Nesse ponto, a coluna foi pesada e, por diferença, obtida a massa de água contida na amostra, que correspondeu ao vol ume-poro para $600 \mathrm{~g}$ desolo. Obteve-seo vol umeporo de $175 \mathrm{~mL}$, que constituiu a unidade para a percolação dos diversos volumes-poros através das colunas.

\section{Percolação}

Depois de saturadas, as col unas foram colocadas em suportes para mantêlas na posição vertical. A seguir, exceção para a testemunha, utilizando um funil, que era mantido em contínua movimentação, colocaram-se em 5 colunas individuais $150 \mathrm{~g}$ do resíduo ácido de forma que a camada sobre a coluna de terra fosse de $5 \mathrm{~cm}$, aproximadamente. Após, iniciou-se o processo de lixiviação deixando-se verter, vagarosamente, água deionizada ajustada em $\mathrm{pH} 4,5$, considerando uma chuva ácida. Durante a lixiviação, manteve-se uma altura de col una líquida de $6 \mathrm{~cm}$ acima da superfície do resíduo. As amostras nas col unas foram lixiviadas com 50 volumes-poros, quantidade correspondente à precipitação total na área do aterro num período de 4 anos, sendo o percolado coletado, a cada $175 \mathrm{~mL}$ (correspondente a um volume-poro), e guardado em frascos graduados.

Após cuidadosa remoção do resíduo, as colunas de solo foram retiradas e seccionadas em quatro partes iguais (0-5, 5-10, 10-15 e 15-20 cm), secas ao ar, destorroadas, pulverizadas em al mofariz deágata, homogeneizadas e guardadas em frascos de polietileno para a análise dos metais pesados.

\section{Extração seqüencial e análises químicas}

As amostras do PV e do resíduo (lixiviados e não lixiviados) foram submetidas à extração seqüencial, segundo método deTessier et al. (1979), que utiliza, seqüencialmente, numa mesma amostra, os seguintes extratores: NaOAc 1 mol L-1, ajustado em pH 5 com HOAc, para metais solúveis, trocáveis e associados a carbonatos; $\mathrm{NH}_{2} \mathrm{OH} \mathrm{HCl}$ (cloridrato de hidroxilamina) 0,04 mol L-1, para metais pesados ligados a óxidos Fe e $\mathrm{Mn} ; \mathrm{H}_{2} \mathrm{O}_{2}$ a $300 \mathrm{~g} \mathrm{~L}-1$ ajustado em pH 2 com $\mathrm{HNO}_{3}$, a $85^{\circ} \mathrm{C}$, para metais pesados complexados pela matéria orgânica. A fração residual foi determinada por digestão numa mistura de $\mathrm{HClO}_{4}$ e HF na proporção de 1:5. Os extratos das amostras de terra, de resíduo e dos efluentes foram analisados para $\mathrm{Pb}, \mathrm{Cr}, \mathrm{Cu}, \mathrm{Zn}, \mathrm{Mn}, \mathrm{Ni}$ e $\mathrm{Cd}$, por espectroscopia de absorção atômica, com forno de grafite. $0 \mathrm{pH}$ das amostras de terra e de resíduo foi obtido em água na relação 1:2,5. A concentração de sulfato solúvel em $\mathrm{CaCl}_{2}$ no resíduo foi determinado por turbidimetria, pelo método de Bissani et al. (1995).

\section{RESULTADOS E DISCUSSÃO}

As diferencas entre os teores de metais pesados totais e extraídos, seqüencial mente, no resíduo ácido, antes e depois da lixiviação, encontram-se no quadro 1.

Verificaram-se, em relação ao total das frações, perdas de $58 \%$, para $\mathrm{Ni} ; 56 \%$, para Cd; $52 \%$, para $\mathrm{Zn} ; 40 \%$, para $\mathrm{Mn}$; $10 \%$, para $\mathrm{Cr} ; 8,7 \%$, para $\mathrm{Cu}$, e $7,5 \%$, para $\mathrm{Pb}$. A el evada concentração de sulfato solúvel no resíduo indica ser esse ânion o principal responsável pela mobilização desses metais. Os teores de $\mathrm{Ni}, \mathrm{Cd}, \mathrm{Zn}, \mathrm{Mn}, \mathrm{Cu}$ e $\mathrm{Pb}$ no resíduo e as respectivas perdas desses elementos na fração $\mathrm{NaOAcde}$, respectivamente, $44,81,45,59,87$ e $100 \%$, permitem a predição de contami nação de aqüíferos por aqueles metais. Os resultados obtidos no quadro 1 revelam, pelo menos qual itativamente, que os teores totais de metais pesados no resíduo e a 
solubilidade desse material em NaOAc deverão ser considerados na avaliação dos riscos de poluição de sistemas naturais.

\section{Coluna de terra sem resíduo}

A distribuição dos metais pesados nas colunas de terra sem resíduo mostrou, depois da lixiviação, que os teores totais desses metais mantiveram-se constantes nas quatro camadas (linha tracejada nas figuras de 1 a 5), apresentando as concentrações de $23 \mathrm{mg} \mathrm{kg}^{-1}$, para Zn; 67,5 $\mathrm{mg} \mathrm{kg}^{-1}$, para M n; $15 \mathrm{mg} \mathrm{kg}^{-1}$, para $\mathrm{Ni}$; 1,0 $\mathrm{mg} \mathrm{kg}^{-1}$, para Cd; 4,4 $\mathrm{mg} \mathrm{kg}^{-1}$, para Cu; $15 \mathrm{mg} \mathrm{kg}^{-1}$, para $\mathrm{Pb}$, e $25 \mathrm{mg} \mathrm{kg}^{-1}$, para $\mathrm{Cr}$.

\section{Coluna de terra com resíduo}

0 movimento das diferentes formas de $\mathrm{Pb}, \mathrm{Cr}$ e Cu nas colunas de terra com a camada do resíduo ácido depois da lixiviação e os respectivos diagramas de barra, mostrando a distribuição percentual relativa daquelas frações aparecem na figura 1. Observa-se translocação daqueles metais que se concentraram, exceção do $\mathrm{Cr}$, na camada superficial $(0-5 \mathrm{~cm})$ da amostra do solo. Esses incrementos relativos à testemunha foram de $255 \mathrm{mg} \mathrm{kg}^{-1}$, para $\mathrm{Pb}$; $80 \mathrm{mg} \mathrm{kg}^{-1}$, para $\mathrm{Cr}$, e 3,6 mg kg-1, para Cu. Tais incrementos corresponderam a $90 \%$ do mobilizado (perda) do resíduo, aproximadamente (Quadro 1). A redução na concentração desses el ementos na camada de resíduo, após a percolação, demonstra que o enriquecimento do solo das colunas, com esses metais, ocorreu pela solubilização no resíduo, e não pelo movimento de partículas finas do resíduo nos poros da coluna de terra.

A distribuição percentual do $\mathrm{Pb}$ nas diferentes formas químicas (Figura 1d) mostra que, na camada superficial, $76 \%$ do $\mathrm{Pb}$ total aparece associado à fração trocável, $16 \%$ a óxidos-Fe e Mn e $8 \%$ à fase residual. A el evada percentagem da fração trocável

Quadro 1. Diferença entre os teores de $\mathrm{Zn}, \mathrm{Mn}, \mathrm{Ni}, \mathrm{Cd}, \mathrm{Pb}, \mathrm{Cu}$ e $\mathrm{Cr}$ totais e extraídos seqüencialmente, no resíduo ácido da CSN, antes e depois da lixiviação com 50 volumes-poro com água em pH 4,5 (média de 5 repetições)

\begin{tabular}{|c|c|c|c|c|c|c|}
\hline \multirow{2}{*}{$\begin{array}{c}\text { Metais } \\
\text { pesados }\end{array}$} & \multicolumn{5}{|c|}{ Solução extratora } & \multirow{2}{*}{$\begin{array}{c}\text { Total das } \\
\text { frações }\end{array}$} \\
\hline & Condições & NaOAc & $\mathrm{NH}_{2} \mathrm{OH} \mathrm{HCl}$ & $\mathrm{H}_{2} \mathrm{O}_{2}+\mathrm{HNO}_{3}$ & $\mathrm{HF}+\mathrm{HClO}_{4}$ & \\
\hline & & & & $-\mathrm{mg} \mathrm{kg}^{-1}$ & & - \\
\hline $\mathrm{Zn}$ & $\begin{array}{l}\text { Resíduo(1) } \\
\text { Resíduo( } \\
\text { Perda(3) }\end{array}$ & $\begin{array}{c}196,87(23)^{(4)} \\
0,00(0) \\
196,87(45)\end{array}$ & $\begin{array}{r}124,37(15) \\
42,00(10) \\
32,37(19)\end{array}$ & $\begin{array}{r}31,25(4) \\
31,25(8) \\
0,00(0)\end{array}$ & $\begin{array}{l}487,51(58) \\
331,75(82) \\
155,76(36)\end{array}$ & $\begin{array}{l}840,00 \\
405,00 \\
435,00\end{array}$ \\
\hline $\mathrm{Mn}$ & $\begin{array}{l}\text { Resíduo } \\
\text { Resíduo } \\
\text { Perda }\end{array}$ & $\begin{array}{c}3788,75(28) \\
510,00(6) \\
3278,75(59)\end{array}$ & $\begin{array}{r}1595,00(12) \\
955,00(12) \\
640,00(12)\end{array}$ & $\begin{array}{l}465,00(3) \\
340,00(4) \\
125,00(2)\end{array}$ & $\begin{array}{l}7917,25(57) \\
6437,50(78) \\
1479,75(27)\end{array}$ & $\begin{array}{r}13766,00 \\
8242,50 \\
5523,50\end{array}$ \\
\hline $\mathrm{N} \mathrm{i}$ & $\begin{array}{l}\text { Resíduo } \\
\text { Resíduo } \\
\text { Perda }\end{array}$ & $\begin{array}{r}480,00(30) \\
80,00(12) \\
400,00(44)\end{array}$ & $\begin{array}{c}180,00(11) \\
43,00(6) \\
137,00(15)\end{array}$ & $\begin{array}{r}37,50(2) \\
30,00(4) \\
7,50(1)\end{array}$ & $\begin{array}{l}877,50(57) \\
514,00(78) \\
363,50(40)\end{array}$ & $\begin{array}{r}1575,00 \\
667,00 \\
908,00\end{array}$ \\
\hline $\mathrm{Cd}$ & $\begin{array}{l}\text { Resíduo } \\
\text { Resíduo } \\
\text { Perda }\end{array}$ & $\begin{array}{l}2,50(46) \\
0,00(0) \\
2,50(81)\end{array}$ & $\begin{array}{l}0,75(14) \\
0,25(10) \\
0,50(16)\end{array}$ & $\begin{array}{l}0,25(4) \\
0,15(6) \\
0,10(3)\end{array}$ & $\begin{array}{l}2,00(36) \\
2,00(84) \\
0,00(0)\end{array}$ & $\begin{array}{l}5,50 \\
2,40 \\
3,10\end{array}$ \\
\hline $\mathrm{Pb}$ & $\begin{array}{l}\text { Resíduo } \\
\text { Resíduo } \\
\text { Perda }\end{array}$ & $\begin{array}{c}1425,00(40) \\
1155,00(35) \\
270,00(100)\end{array}$ & $\begin{array}{c}675,00(19) \\
675,00(20) \\
0,00(0)\end{array}$ & $\begin{array}{r}121,25(3) \\
121,25(4) \\
0,00(0)\end{array}$ & $\begin{array}{c}1378,75(38) \\
1378,75(41) \\
0,00(0)\end{array}$ & $\begin{array}{r}3600,00 \\
3330,00 \\
270,00\end{array}$ \\
\hline $\mathrm{Cu}$ & $\begin{array}{l}\text { Resíduo } \\
\text { Resíduo } \\
\text { Perda }\end{array}$ & $\begin{array}{l}5,00(11) \\
1,50(4) \\
3,50(87)\end{array}$ & $\begin{array}{l}1,70(4) \\
1,50(4) \\
0,20(5)\end{array}$ & $\begin{array}{c}19,75(43) \\
18,79(46) \\
0,30(8)\end{array}$ & $\begin{array}{c}19,63(42) \\
19,63(46) \\
0,00(0)\end{array}$ & $\begin{array}{r}46,08 \\
41,66 \\
4,00\end{array}$ \\
\hline $\mathrm{Cr}$ & $\begin{array}{l}\text { Resíduo } \\
\text { Resíduo } \\
\text { Perda }\end{array}$ & $\begin{array}{l}0,00(0) \\
0,00(0) \\
0,00(0)\end{array}$ & $\begin{array}{r}12,50(1) \\
12,50(2) \\
0,00(0)\end{array}$ & $\begin{array}{r}37,50(4) \\
37,50(5) \\
0,00(0)\end{array}$ & $\begin{array}{c}823,33(95) \\
735,00(93) \\
83,33(100)\end{array}$ & $\begin{array}{r}873,33 \\
785,00 \\
88,33\end{array}$ \\
\hline
\end{tabular}

(1) Teores dos metais no resíduo antes do processo de lixiviação; ${ }^{(2)}$ Teores dos metais no resíduo depois do processo de lixiviação;

(3) Perda do metal no processo de lixiviação; ${ }^{(4)}$ Percentagem do metal na fração em relação ao total das frações do metal no resíduo. 
pode ser atribuída ao pequeno tempo de interação solução percolante $x$ solo (uma semana), não permitindo que os metais ligados a óxidos-Fe e Mn e, ou, na fase residual, mais estáveis, predominassem.

Amaral Sobrinho et al. (1997) verificaram a passagem das formas mais solúveis para as de menor solubilidade depois de 18 meses de incubação resíduo x solo.

$\mathrm{Nãose}$ podedescartar a possibilidade de o $\mathrm{NaOAC}$ $1 \mathrm{~mol} \mathrm{L-1} \mathrm{pH} \mathrm{5,0} \mathrm{ter} \mathrm{retirado} \mathrm{Pb} \mathrm{ligado} \mathrm{a} \mathrm{óxidos-Fee}$ Mn (McLaren \& Crawford, 1973; Tessier et al., 1979). As distribuições percentuais de $\mathrm{Cr}$ e $\mathrm{Cu}$ em cada fração e com a profundidade (Figuras le e 1f) ocorreram apenas nas formas óxido e residual. Nas camadas superficiais, para $\mathrm{Cr}$ e para $\mathrm{Cu}$, houve um acréscimo de, aproximadamente, $25 \%$ da fração óxido. As associações de $\mathrm{Cr}$ eCu com as frações óxidoFe e $\mathrm{Mn}$ e residual, observadas neste ensaio, corroboram com os resultados de Amaral Sobrinho (1993) queverificou, em amostras desse mesmo solo incubadas com o resíduo ácido, uma el evada afinidade desses metais pelas frações óxido-FeeMn e residual. Resultados semel hantes foram encontrados, para $\mathrm{Cr}$, por Chang et al. (1984), Canãdas et al. (1986) e McGrath \& Lane (1989) e, para $\mathrm{Cu}$, por Korte et al. (1976), Biddappa et al. (1982) e Davis et al. (1988).

A ausência de metais pesados na fração orgânica, principalmente $\mathrm{Pb}$, pelas suas el evadas perdas durante a lixiviação (Quadro 1), pode ser atribuída ao baixo teor de matéria orgânica no sol o utilizado.

As figuras 2a, 3a, 4a e 5a mostram para Zn, Mn, $\mathrm{Ni}$ e Cd, respectivamente, depois do processo de lixiviação, os teores totais e os extraídos, seqüencial mente, nas quatro camadas da coluna de terra. Verificam-se, em todas elas, incrementos das concentrações totais, em relação à testemunha, que
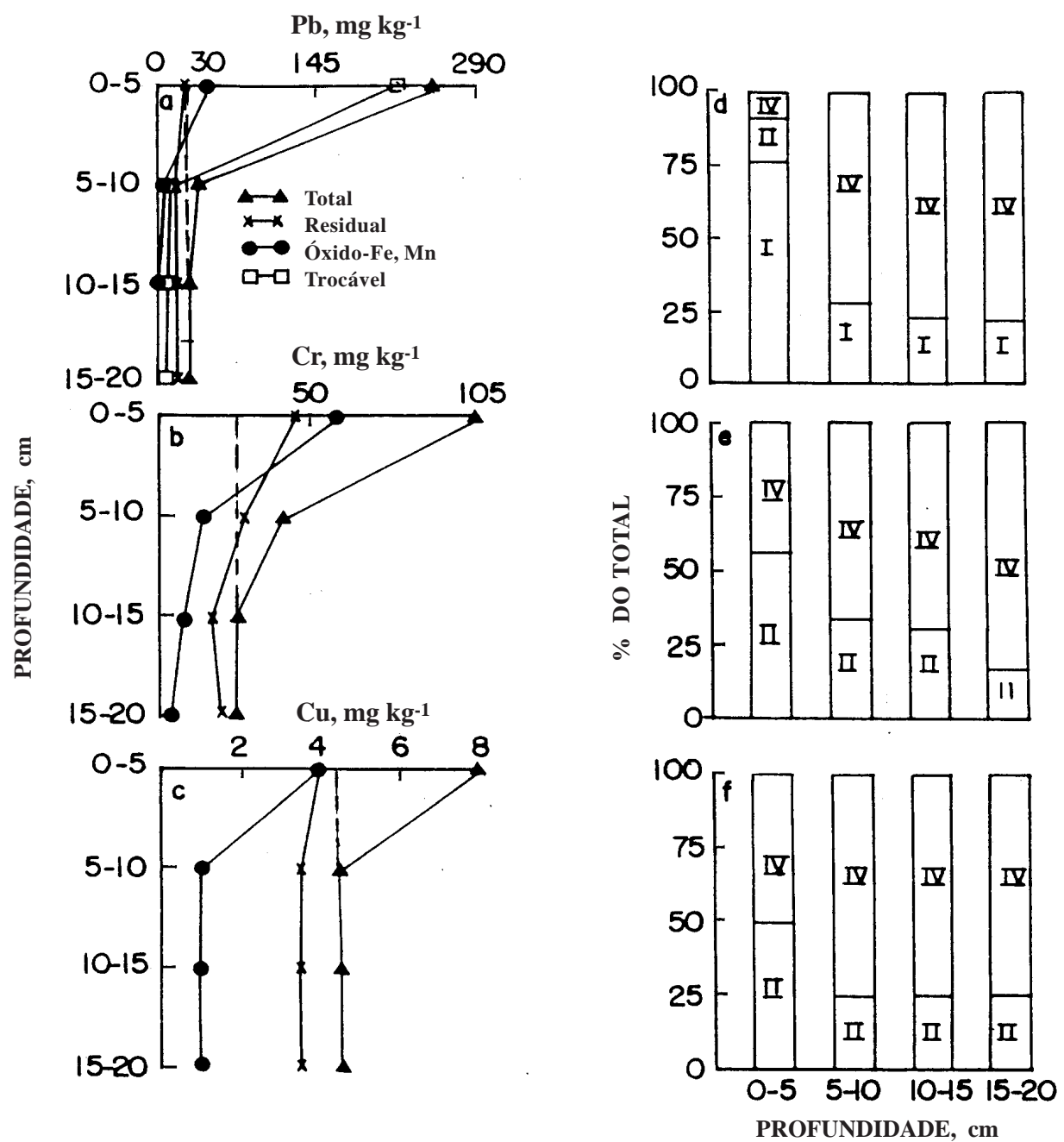

Figura 1. Movimento das diferentes formas químicas de $\mathbf{P b}$ (a), $\mathbf{C r}$ (b) e Cu (c) nas colunas contendo a pilha do resíduo ácido e os respectivos diagramas de barra mostrando a distribuição percentual relativa: Trocável (I), Óxido-Fe,Mn (II), Orgânica (III) e Residual (IV), de Pb (d), Cr (e) e Cu (f) após processo de lixiviação com 50 volumes-poro com água pH 4,5. 

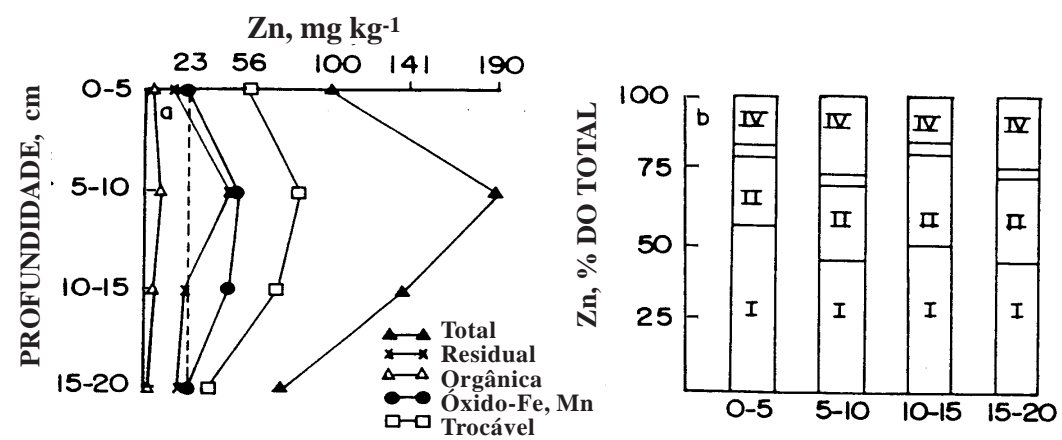

PROFUNDIDADE, $\mathrm{cm}$

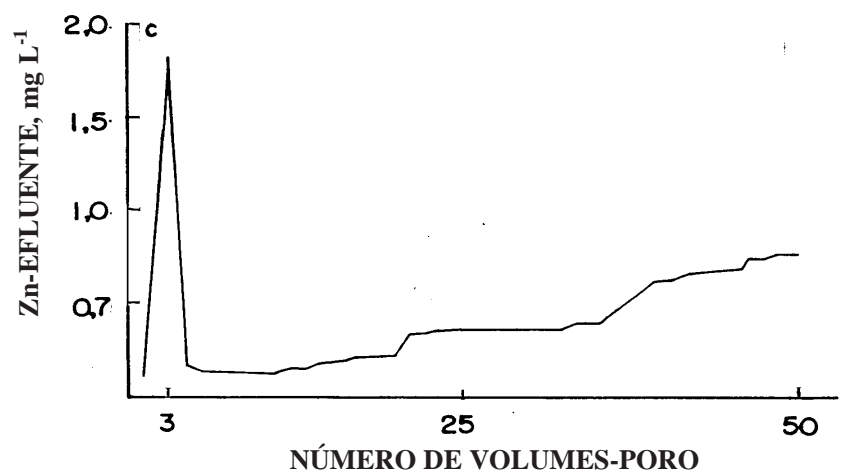

Figura 2. Movimento das diferentes formas químicas de $\mathrm{Zn}$ nas colunas contendo a pilha do resíduo ácido (a), os respectivos diagramas de barra mostrando a distribuição percentual relativa de $\mathrm{Zn}$ : Trocável (I), Óxido-Fe e Mn (II), Orgânica (III) e Residual (IV), após processo de lixiviação com 50 volumes-poros com água pH 4,5 (b) e os teores nos efluentes (c).
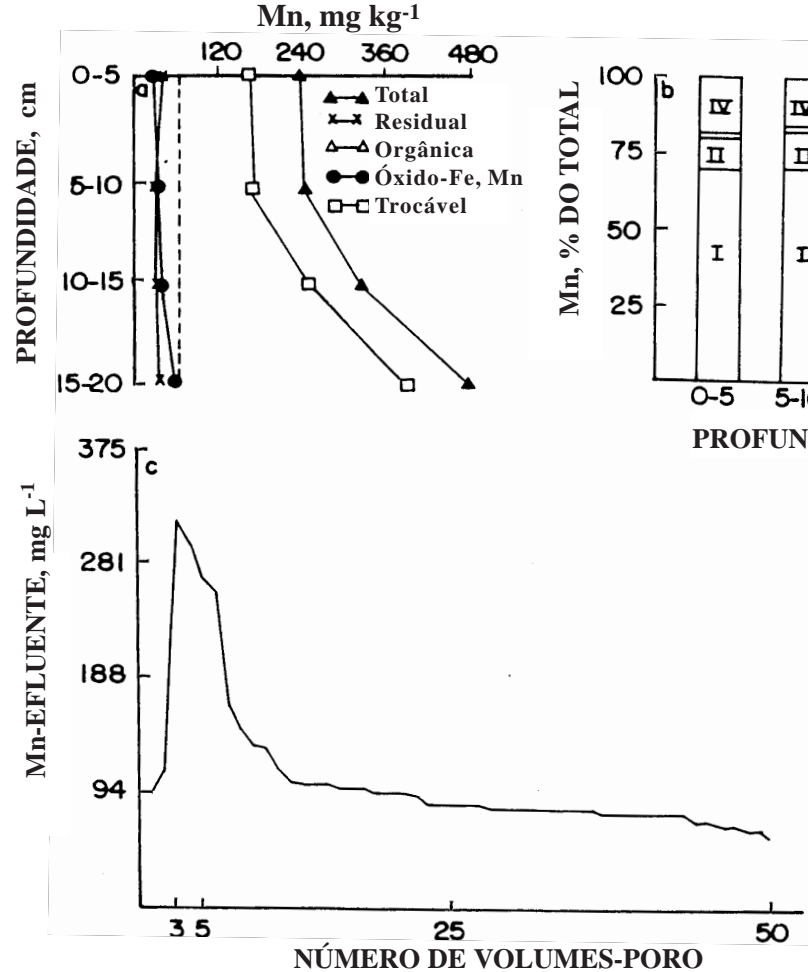

NÚMERO DE VOLUMES-PORO

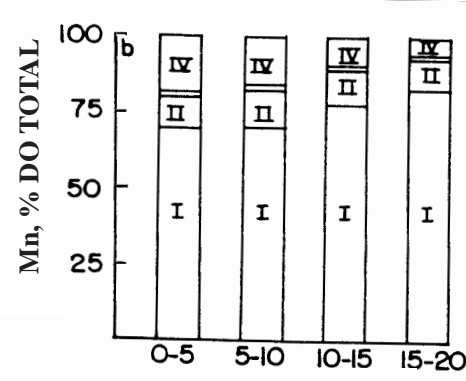

PROFUNDIDADE, cm

Figura 3. Movimento das diferentes formas químicas de Mn nas colunas contendo a pilha do resíduo ácido (a), os respectivos diagramas de barra mostrando a distribuição percentual relativa de Mn: Trocável (I), Óxido-Fe e Mn (II), Orgânica (III) e Residual (IV), após processo de lixiviação com 50 volumes-poros com água pH 4,5 (b) e os teores nos efluentes (c). 


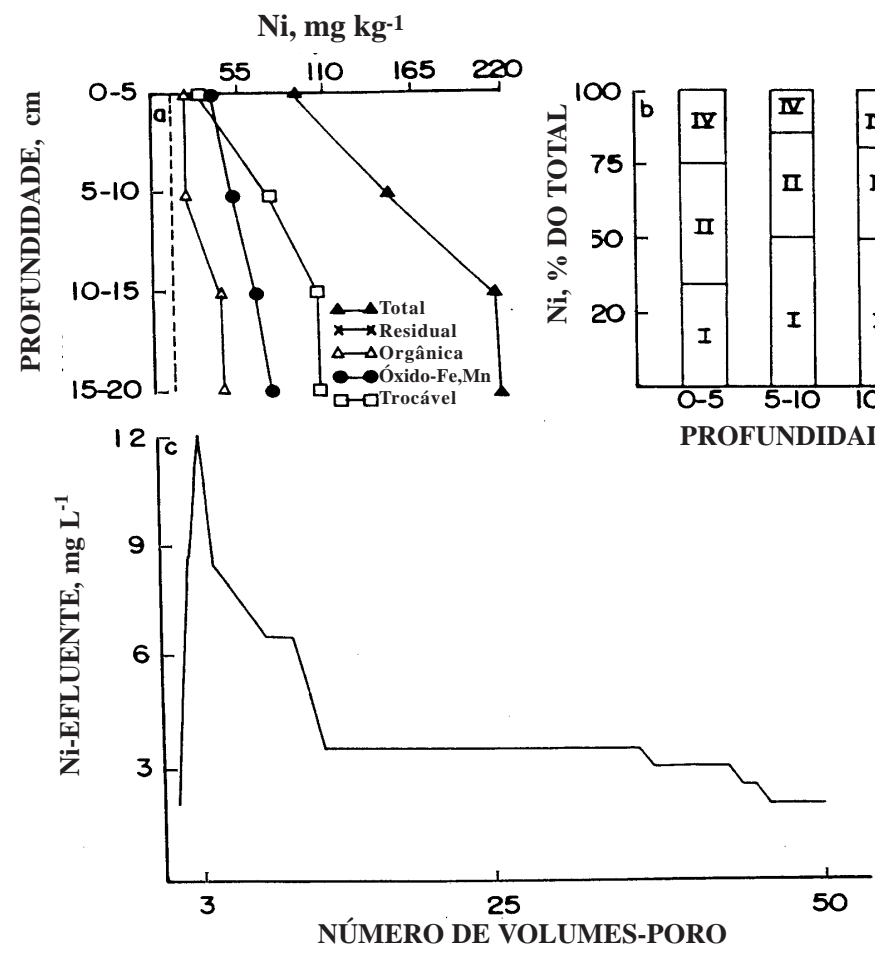

Figura 4. Movimento das diferentes formas químicas de Ni nas colunas contendo a pilha do resíduo ácido (a), os respectivos diagramas de barra mostrando a distribuição percentual relativa de $\mathrm{Ni}$ : Trocável (I), Óxido-Fe,Mn (II), Orgânica (III) e Residual (IV), após processo de lixiviação com 50 volumesporos com água pH 4,5 (b) e os teores nos efluentes (c).
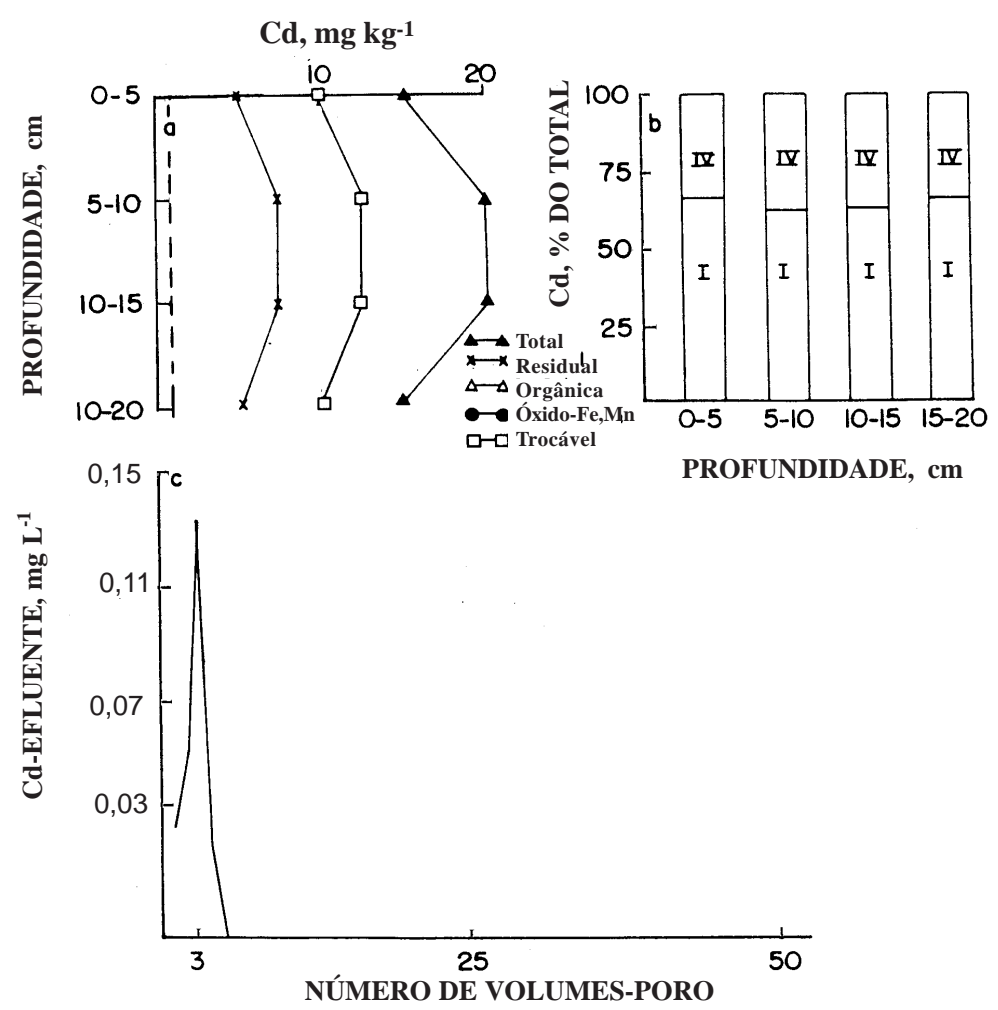

Figura 5. Movimento das diferentes formas químicas de $\mathrm{Cd}$ nas colunas contendo a pilha do resíduo ácido (a), os respectivos diagramas de barra mostrando a distribuição percentual relativa de Cd: Trocável (I), Óxido-Fe,Mn (II), Orgânica (III) e Residual (IV), após processo de lixiviação com 50 volumes-poros com água pH 4,5 (b) e os teores nos efluentes (c). 
atingiram os valores máximos de $164 \mathrm{mg} \mathrm{kg}^{-1}$, para Zn (2a camada); $203 \mathrm{mg} \mathrm{kg}^{-1}$, para Ni (última camada); $409 \mathrm{mg} \mathrm{kg}^{-1}$, para Mn (última camada), e $10 \mathrm{mg} \mathrm{kg}^{-1}$, para Cd (2a e 3a camadas). Do total mobilizado do resíduo (Quadro 1), observou-se, na coluna de terra, retenção de $94 \%$ para Zn, $68 \%$ para $\mathrm{Ni}, 18 \%$ para $\mathrm{Mn}$ e $97 \%$ para $\mathrm{Cd}$. Tais percentuais e os relativamente altos teores desses metais no resíduo predizem el evadíssimos potenciais de contaminação de aqüíferos.

No Estado do Rio de J aneiro, as concentrações máximas permitidas para lançamento de efluentes em corpos d'água são de 1 mg L-1 para Zn, Ni e Mn e de 0,1 mg L-1 para Cd (FEEMA, 1985). As figuras 2c a 5 c mostram que as maiores concentrações dos metais pesados nos efluentes das colunas aconteceram no $3^{\circ}$ volume-poro, decrescendo, drasticamente, depois. Para Zn (Figura 2c) e Cd (Figura 5c), os limites estabelecidos pela FEEMA foram levemente ultrapassados no $3^{\circ}$ volume-poro. Para $\mathrm{Ni}$ e Mn, as concentrações dos efluentes foram el evadas. Para Ni (Figura 4c), verificou-se um pico máximo de $12 \mathrm{mg} \mathrm{L-1}$ no $3^{\circ}$ volume-poro, daí decrescendo até o final do experimento, onde a sua concentração (2 mg L-1) era, ainda, superior ao limite permitido. Para Mn (Figura 3c), a elevada concentração de $317 \mathrm{mg} \mathrm{L}^{-1}$ no $3^{\circ}$ volume-poro, diminuindo para $60 \mathrm{mg} \mathrm{L}^{-1}$ no final do experimento, indica, demodo inequívoco, o el evado potencial tóxico do resíduo ácido da CSN.

As distribuições percentuais de $\mathrm{Zn}, \mathrm{Ni}, \mathrm{Mn}$ e $\mathrm{Cd}$ nas diferentes formas químicas, nas quatrocamadas, aparecem nas figuras $2 \mathrm{~b}$ a $5 \mathrm{~b}$. Para Zn e Ni, a fração trocável situou-seem torno de $50 \%$ e, para M n eCd, próximo de $70 \%$, números que mais uma vez alertam para a elevada labilidade e/ou biodisponibilidade. El levadas percentagens de metais pesados associados à fração trocável foram observadas, para Zn, Ni e Cd, em solos do J apão contaminados por resíduos de fundição (Biddappa et al., 1982); para Zn (Dowdy \& Volk, 1983) e Ni (Petruzzelli et al., 1986), em ensaios de colunas de solo tratados com lodo de esgoto sól ido, e para Mn (Sims et al., 1979 eShumam, 1985), após a aplicação de sais solúveis.

As correl ações entrea concentração total do metal e a respectiva fração extraída com $\mathrm{NaOAc}(\mathrm{Zn}$ : $r=0,95 * *, \mathrm{Ni}: r=0,99 * *, \mathrm{Mn}: r=0,99 * *$ e para $\mathrm{Cd}$ : $r=0,99 * *)$, mostram a possibilidade de se usar tal extrator, pelo menos nos estudos de previsão de contaminação e movimentação de metais em solos que receberam resíduos siderúrgicos ácidos.

\section{CONCLUSÕES}

Considerando que: (a) nas condições normais do ARSI não haveria fluxo contínuo de água através das pilhas de resíduos; (b) somente uma fração da precipitação pluviométrica local percolaria através dos resíduos ese infiltraria no solo; (c) a alternância de períodos secos e chuvosos possibilitaria a formação de compostos mais estáveis de Zn, Mn, Ni, $\mathrm{Cd}, \mathrm{Cr}, \mathrm{Pb}$ e $\mathrm{Cu}$; (d) a disposição das camadas dos resíduos no ARSI apresentaria um diâmetro maior na base, dificultando a percolação da água; (e) este estudo desimulação doARSI em laboratório, através do uso de colunas de solo, foi qualitativo e um tanto drástico, acredita-se que as seguintes conclusões possam ser emitidas.

1. Os dados obtidos neste trabal ho salientam a importância da concentração total de metais no resíduo e de suas solubilidades em NaOAc na avaliação dos riscos de contaminação provocados pela disposição final de resíduos siderúrgicos em aterros.

2. Os percentuais de retenção observados nas colunas, as relativamente el evadas concentrações desses metais no resíduo e as concentrações de $\mathrm{Mn}$ eNi nos efluentes das colunas superiores aos padrões permitidos pela FEEMA predizem elevadíssimos potenciais de contaminação de aqüíferos.

3. No resíduo ácido, el evadas concentrações de $\mathrm{Pb}, \mathrm{Cu}$ e Cr ocasionaram a mobilização desses metais para os primeiros $5 \mathrm{~cm}$ da coluna de solo.

4. $\mathrm{Zn}, \mathrm{Mn}, \mathrm{Ni}$ e $\mathrm{Cd}$, nas colunas contendo o resíduo, apresentaram o mesmo padrão de distribuição, isto é, aumento da concentração com a profundidade.

\section{LITERATURA CITADA}

ALLOWAY, B.J. The origins of heavy metals in soils. In: ALLOWAY, B.J. Heavy metals in soils. New York, J ohn Wiley, 1990. p.29-39.

AMARAL SOBRINHO, N.M.B. Interação dos metais pesados de resíduos siderúrgi cos com solo Podzól ico Vermel ho-Amarelo. Viçosa, Universidade Federal de Viçosa, 1993. 163p. (Tese de Doutorado)

AMARAL SOBRINHO, N.M.B.;VELLOSO,A.C.X.\& OLIVEIRA, C. Solubilidade de metais pesados em solo tratado com resíduo siderúrgico. R. Bras. Ci. Solo, 21:9-16, 1997.

ASSOCIAÇÃO BRASILEIRA DE NORMASTÉCNICAS - ABNT. Amostragem de resíduos sólidos. São Paulo, 1985. 25p. Projeto 1.63.02-004

BIDDAPPA, C.C.; CHINO, M. \& KUMAZAWA, K. Migration of heavy metals in two japanese soils. Plant Soil, 66:299-316, 1982.

BISSANI, C.A.; KÄMPF, N. \& LUZ, P.C.R. Determinação de sulfato solúvel em solos tiomórficos de áreas de mineração de carvão. In:CONGRESSO BRASILEIRO DE CIÊNCIA DO SOLO, 25., Viçosa, 1995. Resumos. Viçosa, Sociedade Brasileira de Ciência do Solo, 1995. p.1535-1537.

CANÃDAS, R.C.; SANCHIDRIAN, J.R. \& RIVERO, V.C. Distribucion de $\mathrm{Pb}, \mathrm{Cd}$, Cu y $\mathrm{Cr}$ entre distintas fases sólidas en algunos tipos de suelos. An. Edafol. Agrobiol., 45:613630, 1986. 
CANTINI, V.L. \& SOUZA, L.F. Aplicações e mercado de rejeitos siderúrgicos da Companhia Siderúrgica Nacional. In: SEMINÁRIO DE RECUPERAÇÃO DE REJEITOS DA INDÚSTRIA METALÚRGICA, 1., Volta Redonda, 1989. Anais. Volta Redonda, CORENE/ABM, 1989. p.131-144.

CHANG, A.C.; PAGE, A.L.; WARNEKE, J.E. \& GRUREVIC, E. Sequential extraction of soil heavy metals in sewage following a sludge aplication. J. Environ. Qual., 13:33-37, 1984.

COMPANHIA BRASILEIRA DE PROJ ETOS INDUSTRIAIS COBRAPI. Relatório básico do plano de proteção ambiental - aterro de resíduos sól idos industriais. Volta Redonda, 1986. $52 \mathrm{p}$.

DAVIS, R.D.; CARLTON-SMITH, C.H.; STARK, J.H. \& CAMPBELL, J.A. Distribution of metals in grassland soils following surface applications of sewage sludge. Environ. Pollut., 49:99-115, 1988.

DOWDY, R.H. \& VOLK, V.V. Movement of heavy metals in soils. In: NELSON, D.W.; ELRICK, D.E. \& TANGI, K.K., eds. Chemical mobility and reactivity in soil systems. Madison, Soil Science Society of America, 1983. p.229-240. (SSSA Special Publication, 11)

FUNDAÇÃO ESTADUAL DE ENGENHARIA DO MEIO AMBIENTE - FEEMA. Padrões de lançamento em corpos receptores, NT 202. Rio de J aneiro, 1985. 20p

KHAN, D.H. \& FRANKLAND, B. Effects of cadmium and lead on radish plants with particular reference to movement of metals through soil profile and plant. Plant Soil, 70:335345, 1983.

KORTE, N.E.; SKOPP, J .; FULLER, W.H.; NIEBLA, E.E. \& ALESII, B.A. Trace element movement in soils: Influence of soil physical and chemical properties. Soil Sci., 122:350359, 1976.
McGRATH, S.P. \& LANE, P.W. An explanation for the apparent losses of metals in a long-term field experiment with sewage sludge. Environ. Pollut., 60:235-256, 1989.

MCLAREN, R.G. \& CRAWFORD, D.V. Studies on soil copper: I. The fractionation of copper in soils. J. Soil Sci., 24:172181, 1973.

PETRUZZELLI, G.; GUIDI, G. \& LUBRANO, L. Modification of heavy metals solubility in soil treated with sewage sludge. In: Processing and use of organic sludge and liquid agricultural wastes. Dordrecht, P.G. Hermite Reidel Pub. Com., 1986. p.478-484.

SHEPPARD, M.I \& \& THIBAULT, D.H. Desorption and extraction of selected heavy metals from soils. Soil Sci. Soc. Am. J., 56:415-423, 1992.

SHUMAM, L.M. Fractionation method for soil microelements. Soil Sci., 140:11-22, 1985.

SIMS, J .L.; DUANGPATRA, P.; ELLIS, J.H. \& PHILLIPS, R.E. Distribution of available manganese in Kentucky soils. Soil Sci., 127:270-274, 1979.

TESSIER, A.; CAMPBELL, P.G.C. \& BISSON, M. Sequential extraction procedure for the speciation of particulate trace metals. Anal. Chem., 51:844-851, 1979.

WELCH, J.E. \& LUND, L.J. Zinc movement in sewage-sludge treated soils as influenced by soil properties, irrigation water quality, and soil moisture level. Soil Sci., 147:208214, 1989.

WILLIAMS, D.E.; VLAMIS, J.; PUKITE, A.H. \& COREY, J.E. Metal movement in sludge treated soils after six years of sludge addition: 2. Nickel, Cobalt, Iron, Manganese, Cromium, and Mercury. Soil Sci., 140:120-125, 1985. 